\title{
Wi-Fi 2.0: Price and Quality Competitions of Duopoly Cognitive Radio Wireless Service Providers with Time-varying Spectrum Availability
}

\author{
Hyoil Kim \\ IBM T. J. Watson Research Center, Hawthorne, NY \\ Email: kimhyoil@us.ibm.com
}

\author{
Jaehyuk Choi and Kang G. Shin \\ EECS Department, University of Michigan, Ann Arbor, MI \\ Email: \{jaehchoi,kgshin\}@eecs.umich.edu
}

\begin{abstract}
The whitespaces (WS) in the legacy spectrum provide new opportunities for the future Wi-Fi-like Internet access, often called Wi-Fi 2.0, since service quality can be greatly enhanced thanks to the better propagation characteristics of the WS than the ISM bands. In the Wi-Fi 2.0 networks, each wireless service provider (WSP) temporarily leases a licensed spectrum band from the licensees and opportunistically utilizes it during the absence of the legacy users. The WSPs in Wi-Fi 2.0 thus face unique challenges since spectrum availability of the leased channel is time-varying due to the ON/OFF spectrum usage patterns of the legacy users, which necessitates the eviction control of in-service customers at the return of legacy users. As a result, to maximize its profit, a WSP should consider both channel leasing and eviction costs to optimally determine a spectrum band to lease and a service tariff. In this paper, we consider a duopoly Wi-Fi 2.0 market where two co-located WSPs compete for the spectrum and customers. The competition between the WSPs is analyzed using game theory to derive the Nash Equilibria (NE) of the price (of the service tariffs) and the quality (of the leased channel, in terms of channel utilization) competitions. The NE existence condition and market entry barriers are also derived. Via an extensive numerical analysis, we show the tradeoffs between leasing/eviction cost, customer arrivals, and channel usage patterns by the legacy users.
\end{abstract}

\section{INTRODUCTION}

The new concept of dynamic spectrum access (DSA) has paved the way for efficient utilization of scarce spectrum resources. DSA enables unlicensed users (called secondary users (SUs)) to opportunistically access under-utilized legacy spectrum bands in the absence of legacy users (called primary users (PUs)). This concept of reusing the legacy spectrum may generate new applications in commercial, public, and military networks, thanks to the recent advances in the software-defined radio (SDR) and cognitive radio (CR) technologies.

The commercial use of DSA has been encouraged by the FCC's ruling released in 2008 [1] that allows unlicensed radio operation in the DTV bands by fixed and portable devices. The fixed devices represent high-power stationary transceivers designed for the last-mile services in rural areas such as IEEE 802.22 [2], and the portable devices represent short-range communication devices in urban areas such as customer terminals for WiFi-like Internet access in spectrum whitespaces (WS), often referred to as Wi-Fi 2.0 [3]-[6]. Of these, Wi-Fi 2.0 is considered as a promising commercial application of DSA with much higher speed and less collision than today's Wi-Fi using the ISM band, thanks to the improved propagation characteristics of the WS such as larger coverage and the wall-penetrating ability of the UHF/VHF bands [7]. ${ }^{1}$

The Wi-Fi 2.0 can be modeled as a three-tier dynamic spectrum market (DSM) [8] consisting of three types of network entity: spectrum license holders, wireless service providers (WSPs), and CR-enabled customers, as illustrated in Fig. 1. The license holders can temporarily lease their spectrum to the WSPs via the multi-winner periodic spectrum auction managed by the spectrum broker (SB). Once a WSP wins a channel at the auction, it provides Internet access to the CR customers (or end-users) by utilizing the leased spectrum, at popular public sites like coffee shops, libraries, or airports. Once a leasing term ends, all channels are returned to the licensees and the WSPs participate in the auction again.

We employ the preemptive spectrum lease model [9] where a channel is modeled as an ON/OFF source [10], [11] as shown in Fig. 2. Here, an OFF period represents spectrum WS during which the lessee of the channel is allowed to access, and an ON period implies PUs' activities during which they can preempt the channel for their own use. Hence, the licensee collects the channel leasing fee only during the OFF periods. In fact, this model is a way to realize the private commons introduced in [8], which is a viable market option to benefit both PUs and SUs by enabling shared channel access while enabling PUs to make extra profit via spectrum leasing.

In this paper, we consider a duopoly Wi-Fi 2.0 network $^{2}$ where two co-located WSPs face price and quality competitions. Each WSP leases a licensed channel with time-varying availability due to the ON-OFF channel usage patterns, and hence upon appearance of PUs the WSP should evict all in-service customers from its network (called channel vacation) to protect the PUs. ${ }^{3}$ We assume the WSP provides the

\footnotetext{
${ }^{1}$ Note that Wi-Fi 2.0 is different from the CR network in ISM bands because the former has to protect incumbent users with priority while the latter can share the spectrum with other types of unlicensed users.

${ }^{2}$ This paper studies duopoly for the ease of analysis, but the duopoly scenario can still provide us enough insight into the network dynamics of Wi-Fi 2.0. The procedures introduced in Sections V and VI can be extended to the multi-WSP case at the expense of the increased number of pricing strategies in Section V-A according to the relative price among WSPs.

${ }^{3}$ Although the WSP can also keep customers in the system while suspending its service during ON periods, it cannot achieve seamless service provisioning.
} 


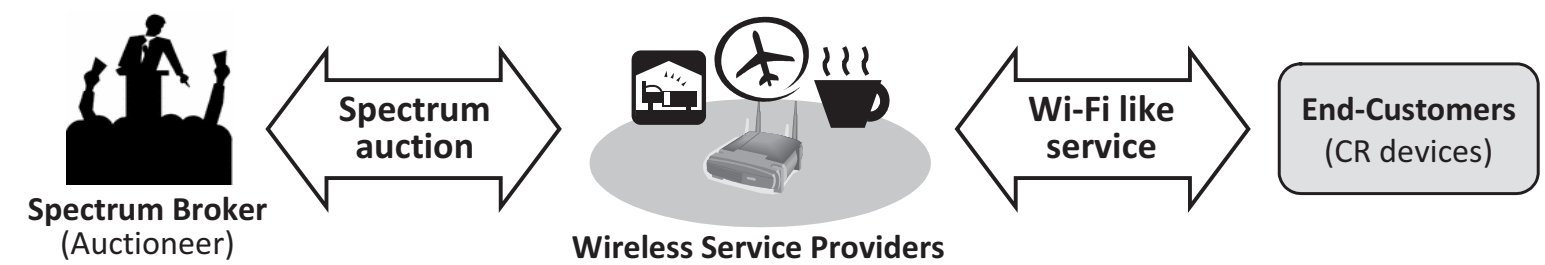

Fig. 1. The three-tier Wi-Fi 2.0 market

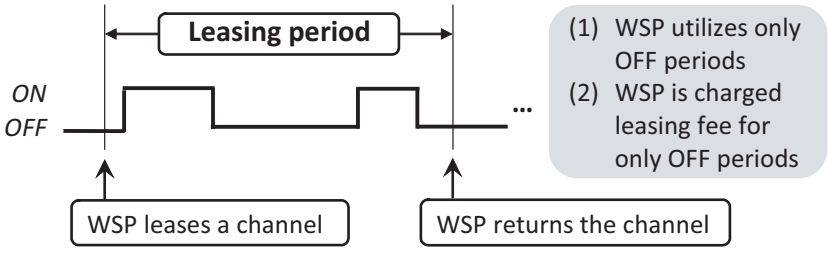

Fig. 2. The preemptible lease model with ON-OFF channels

evicted customers with a monetary compensation by (partially) reimbursing their service charges for the sake of customer satisfaction. Therefore, a WSP should lease a channel with a proper quality in terms of channel utilization by PUs, that incurs less eviction and smaller leasing cost. Each WSP should also determine the optimal price strategy in terms of the service tariff, because a higher price than its competitor will result in less customer arrivals and less profit according to the customers' preference on services. However, the price must be set high enough to be profitable, exceeding the sum of the channel leasing and eviction costs.

Our contribution is three-fold. First, we model the interaction between WSPs as a joint game with price and quality competitions while accounting for time-varying spectrum availability. The existing game-theoretic approaches to the dynamic spectrum market [12]-[14] have been limited to static idle channels, and to the best of our knowledge, this is the first attempt to incorporate the effect of time-varying channel availability in the game-theoretic framework. Next, we model the market dynamics as a Markov chain, and derive the Nash Equilibria (NE) of the price and quality games and the market entry barrier for each WSP. Finally, we perform an extensive numerical analysis to provide insightful results about the market dynamics of the Wi-Fi 2.0 network.

The rest of the paper is organized as follows. Section II briefly reviews related work, and Section III introduces the system model and assumptions used in the paper. Section IV models the problem as a Markov Chain and derives the profit and cost functions. Then, Section V and Section VI formulate and analyze the price and quality competitions, respectively. The market dynamics under various network conditions are shown via an extensive numerical analysis in Section VII, and the paper concludes with Section VIII.

\section{RELATED WORK}

Jia and Zhang [12] studied price and capacity competition in a duopoly DSA market, assuming that the customer arrival rate is determined by a quadratic utility function. However, it may not apply to our case where customers choose a service based on price. Duan et al. [13] studied a similar problem with consideration to physical-layer characteristics of heterogeneous end-users, and derived threshold-type pricing rules. However, they assumed that the spectrum leasing cost is constant and does not depend on the total demand in the spectrum auction. Kasbekar et al. [14] considered a hierarchical game of quantity-price competition, with a two-level prioritized service available to the end-users, and Kasberkar and Sarkar [15] analyzed the case where the availability of licensed channels is determined with some probability while no channel-state transition occurs during a lease term. None of the above work, however, considered time-varying spectrum availability, while assuming that a leased channel is always idle during a leasing term. Although time-varying spectrum availability has been considered by Kim and Shin [9], its focus was customer admission and eviction control at a single WSP, not the market competition between co-located WSPs.

\section{SySTEM MOdEL}

In this section, we describe the system model and assumptions used throughput the paper.

\section{A. Channel Model}

We assume WSP $i(i=1,2)$ leases a channel with capacity $C_{i}$ from the spectrum auction ${ }^{4}$ where each channel is modeled as an ON-OFF source as shown in Fig. 2 while ON and OFF periods are exponentially distributed with rate $\lambda_{i}^{O N}$ and $\lambda_{i}^{O F F}$. Then, channel utilization by PUs, denoted by $u_{i}$, is given as

$$
u_{i}=\frac{1 / \lambda_{i}^{O N}}{\left(1 / \lambda_{i}^{O N}+1 / \lambda_{i}^{O F F}\right)}=\frac{\lambda_{i}^{O F F}}{\left(\lambda_{i}^{O N}+\lambda_{i}^{O F F}\right)} .
$$

This type of channel model has been used in many applications [10], [11], since it can describe PUs' signal activity patterns in a continuous time-domain.

Estimation of the channel parameters (i.e., $\lambda_{i}^{O N}, \lambda_{i}^{O F F}$, and $u_{i}$ ) is possible via spectrum sensing, as discussed in [10]. Detection of the ON/OFF patterns can also be achieved via spectrum sensing, which is outside the scope of this paper.

We assume homogeneous channel capacities such that $C_{i}=$ $C, \forall i$, which would be the case when the same type of licensed bands are considered (e.g., multiple DTV channels).

\section{B. Auction Model}

We consider a multi-winner periodic spectrum auction [16], [17], where WSP $i$ leases a channel with the utilization of $u_{i}$ and pays the leasing fee of $L_{i}$ per unit-time. To describe

\footnotetext{
${ }^{4}$ We reserve $i$ as a WSP index and use $-i$ to denote WSP $i$ 's competitor.
} 


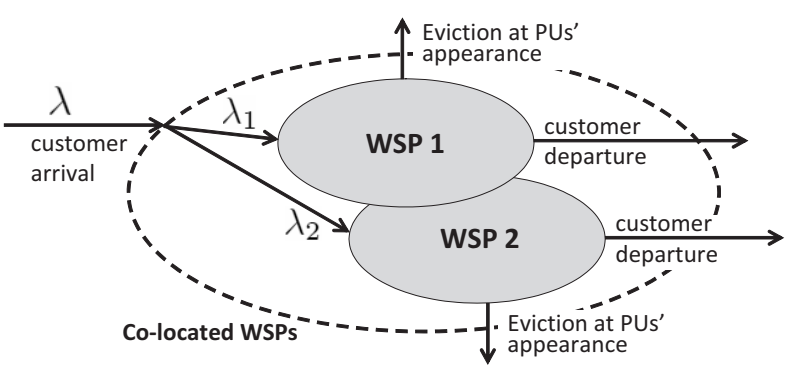

Fig. 3. A duopoly Wi-Fi 2.0 network

the form of $L_{i}$, we introduce the concept of effective channel capacity, denoted by $C_{i}^{e f f}$, given as

$$
C_{i}^{e f f}=\left(1-u_{i}\right) C,
$$

which implies the total effective amount of leased spectrum available for WSP $i$.

Then, the DSA auction model in [12], [18] has shown that the unit price function $l$, i.e., the leasing price per unitbandwidth, is given as

$$
l=\gamma_{1}\left(C_{i}^{e f f}+C_{-i}^{e f f}\right)^{\gamma_{2}}, \gamma_{1}>0, \gamma_{2} \geq 1,
$$

which is a positive, non-decreasing and convex function of $\left(C_{i}^{\text {eff }}+C_{-i}^{\text {eff }}\right)$. That is, the leasing cost depends on the total spectrum demand in the auction market, where $\gamma_{1}$ is the baseline cost when the total demand is unity. In addition, the leasing cost increases faster than proportionally to the total demand (i.e., $C_{i}^{e f f}+C_{-i}^{e f f}$ ) due to the competition between WSPs for the limited spectrum resources auctioned off, where the degree of competition is described by $\gamma_{2}$.

Finally, the leasing cost function $L_{i}$ of WSP $i$ is given as

$$
L_{i}=C_{i}^{e f f} \cdot l=\bar{\gamma}_{1}\left(1-u_{i}\right)\left(2-u_{i}-u_{-i}\right)^{\gamma_{2}},
$$

where $\bar{\gamma}_{1}=\gamma_{1} C^{1+\gamma_{2}}>0$ is the normalized $\gamma_{1}$.

\section{Service Model}

1) Customer arrivals and departures: We assume that customer arrivals follow a Poisson distribution with rate $\lambda$ and their service time is exponentially distributed with mean $1 / \mu$. We define $\rho:=\lambda / \mu$ and assume $0<\rho<1$. In addition, the bulk customer arrivals are split into two flows such that WSP $i$ has arrival rate $\lambda_{i}$ and $\lambda=\lambda_{i}+\lambda_{-i}$ as shown in Fig. 3 .

We assume that each customer demands the bandwidth of $B(B \leq C)$ where $C$ is a multiple of $B$. Then, by defining $\alpha:=C / B(\geq 1)$, which is a positive integer, we can have up to $\alpha$ concurrent in-service customers at a WSP.

2) Service price: An in-service customer at WSP $i$ is charged by $p_{i}$ per unit-time, where it is assumed $\underline{\Pi}_{i} \leq p_{i} \leq \bar{\Pi}$. $\bar{\Pi}$ is referred to as the monopoly price above which WSP $i$ would have no customer arrivals because customers may not choose the 'best-effort' CR service if the 'guaranteed' legacy service offers more competitive price. Therefore, $\bar{\Pi}$ is determined by the tariff of the legacy services (e.g., 3G networks) and is assumed given a priori. On the other hand, $\underline{\Pi}_{i}$ is called the marginal price under which the WSP cannot make profit due to the channel leasing and user eviction cost. $\underline{\Pi}_{i}$ will be derived in Section V.

The monopoly price can also be modeled as qualitysensitive, such that $\bar{\Pi}$ becomes smaller if customers experience more frequent evictions. In such a case, CR WSPs need to lease good-quality channels to maintain larger $\bar{\Pi}$, which is left as our future work.

3) Service discovery and preference: We assume a WSP broadcasts beacons at its leased channel while it is idle (i.e., OFF), to indicate its network is in service. Then, an arriving customer scans a predetermined range of channels (e.g., a list of DTV channels reserved for Wi-Fi 2.0) to find in-service WSPs at its location, and selects the one advertising smallest service price. ${ }^{5}$ In case the WSP chosen by the customer is fully occupied by SUs, the customer is assumed to leave the Wi-Fi 2.0 site. $^{6}$

When a WSP's channel is occupied by PUs (i.e., ON), it cannot broadcast beacons and no customer visits its network. Therefore, even if $p_{i}>p_{-i}$, WSP $i$ can have arrivals while WSP $-i$ 's channel is busy. In summary, when $p_{i}>p_{-i}$, (1) $\lambda_{i}=0$ and $\lambda_{-i}=\lambda$ while WSP $-i$ 's channel is idle, and (2) $\lambda_{i}=\lambda$ and $\lambda_{-i}=0$ while WSP $-i$ 's channel is busy and WSP $i$ 's channel is idle. In addition, when $p_{i}=p_{-i}$, we have $\lambda_{i}=\lambda_{-i}=\lambda / 2$ while both WSPs have idle channels.

4) User eviction: At appearance of PUs, a WSP should evict all in-service customers from the network to protect the PUs. ${ }^{7}$ Each evicted user is compensated by a reimbursement of $I$, where $I=\beta \cdot p_{i} / \mu, \beta>0$, i.e., $\beta$ times the average service charge without eviction. We also assume $\beta \leq 1$ to make the compensation upper-bounded by what customers pay on average. In addition, we assume $\lambda_{i}^{O F F} / \mu=(1 / \mu) /\left(1 / \lambda_{i}^{O F F}\right)<$ 1 , because it is not beneficial to lease a channel that cannot serve even a single session in an OFF period.

It is also possible to differentiate the reimbursement rate $\beta$ between WSPs such as $\beta_{i}$ where the service tariff is described by $\left(p_{i}, \beta_{i}\right)$. Such an extension, however, incurs higher complexity due to the increased search space as $\left(p_{i}, \beta_{i}, u_{i}\right)$.

\section{Two-Stage Market Competition}

The market competition between WSPs can be modeled as a two-stage game, consisting of price and quality games. The quality game is performed periodically at every auction, where each WSP competes for the desired quality of spectrum resources to lease, in terms of $u_{i}$. The quality game is a oneshot game, and thus a WSP cannot return or change the leased channel during a leasing term. During a leasing term, two WSPs perform a price competition to determine the optimal price $p_{i}$ for their maximal profit. The quality game is also called a full-game in the sense that the optimal quality of

\footnotetext{
${ }^{5}$ One can consider other factors in service preference including QoS, data rate, and channel quality. In this paper, we focus on the price as a sole factor.

${ }^{6}$ This is a reasonable assumption since the WSP-customer relationship is volatile due to the flexible design of CR devices [6]. That is, CR customers may choose different services (e.g., Wi-Fi, 3G networks) by reconfiguring themselves if the desired WSP's service is not instantly available.

${ }^{7} \mathrm{We}$ assume the evicted customers will use alternative services such as Wi-Fi or $3 \mathrm{G}$ networks, due to the flexibility of the CR devices.
} 


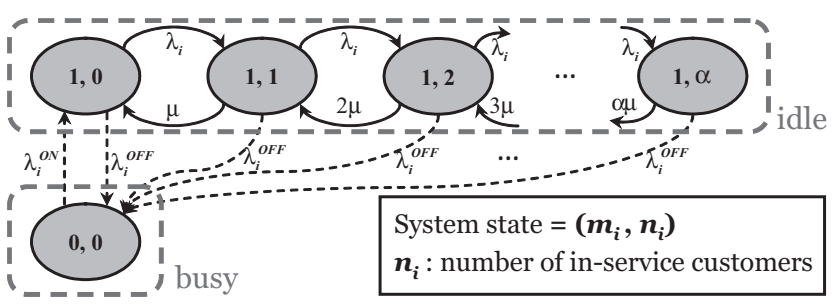

Fig. 4. State transition of WSP $i$ 's system

spectrum is determined by assuming the NE prices of the two WSPs achieved at a price $s u b$-game.

As the main objective of a WSP is to maximize its profit, the profit function must be analytically derived before investigating the price and quality competition. To derive the profit, we define the system state of WSP $i$ as $s_{i}=\left(m_{i}, n_{i}\right)$ where $m_{i}$ is the channel state such that $m_{i}=0$ if channel is busy and $m_{i}=1$ otherwise, and $n_{i}$ is the number of inservice customers with $n_{i} \in\left[0, m_{i} \alpha\right]$. Then, the system can be modeled as a Markov Chain under the assumption of Poisson arrivals, exponential service times, and exponential $\mathrm{ON}$ and OFF periods.

Fig. 4 illustrates the state-transition diagram of the Markov Chain. The horizontal transitions represent the state transitions by the customer arrivals and departures, and the vertical transitions represent the state transitions due to ON-OFF channel state changes. A customer arrival is accepted by the system if $n_{i}<m_{i} \alpha$. When an idle channel becomes busy, all $n_{i}$ customers are evicted from the system.

From Fig. 4, we first notice that

$$
\begin{aligned}
\pi_{0,0} & =u_{i}, \\
\sum_{n_{i}=0}^{\alpha} \pi_{1, n_{i}} & =1-u_{i},
\end{aligned}
$$

where $\pi_{s_{i}}=\pi_{m_{i}, n_{i}}$ denotes the stationary distribution of the system. Then, the global balance equations can be derived in a matrix form as follows.

$$
\mathbf{A}\left(\pi_{1,0} \pi_{1,1} \ldots \pi_{1, \alpha}\right)^{\mathrm{T}}=\left(\begin{array}{llll}
u \cdot \frac{\lambda_{i N}^{O N}}{\mu} & 0 & \ldots & 0
\end{array}\right)^{\mathrm{T}},
$$

where

$$
\mathbf{A}=\left(\begin{array}{cccccccc}
f(0) & -1 & 0 & 0 & \ldots & 0 & 0 & 0 \\
-\rho_{i} & f(1) & -2 & 0 & \ldots & 0 & 0 & 0 \\
0 & -\rho_{i} & f(2) & -3 & \ldots & 0 & 0 & 0 \\
\ldots \ldots & \ldots & \ldots & \ldots \ldots & \ldots \ldots \ldots \ldots \ldots \ldots \ldots \\
0 & 0 & 0 & 0 & \ldots & -\rho_{i} & f(\alpha-1) & -\alpha \\
0 & 0 & 0 & 0 & \ldots & 0 & -\rho_{i} & f(\alpha)-\rho_{i}
\end{array}\right),
$$

by defining $f(k):=\rho_{i}+\lambda_{i}^{O F F} / \mu+k$ and $\rho_{i}:=\lambda_{i} / \mu$. Therefore, using Eq.(2) the stationary probability is found as

$$
\left(\begin{array}{llll}
\pi_{1,0} & \pi_{1,1} & \ldots & \pi_{1, \alpha}
\end{array}\right)^{\mathrm{T}}=\mathbf{A}^{-1}\left(\begin{array}{llll}
u \cdot \frac{\lambda_{i}^{O N}}{\mu} & 0 & \ldots & 0
\end{array}\right)^{\mathrm{T}} .
$$

Although a numerical analysis can be used to find $\mathbf{A}^{-1}$, the stationary probability in a closed-form is preferred in analyzing the price and quality games to obtain an insight in the form of the price and quality NEs. Therefore, we approximate the original Markov Chain by applying a state decomposition technique introduced in [19].

According to [19], we can group the states in Fig. 4 with the same $m_{i}$ (i.e., the states in the same row) together as long as the vertical state-transition rates are much smaller than the horizontal state-transition rates. In DSA, this condition is expected to be met in many applications because spectrum reuse is intended for under-utilized channels with relatively longer ON/OFF periods (e.g., TV bands) compared to the customer arrival/departure by the SUs. In Section VII, we will quantify the impact of this approximation on the accuracy of the analysis through extensive numerical experiments.

After the decomposition, the system becomes $M / M / \alpha / \alpha$ while the channel is idle. Hence, we can express $\pi_{s_{i}}$ as

$$
\pi_{s_{i}} \approx \pi_{n_{i} \mid m_{i}} \cdot P\left(m_{i}\right)= \begin{cases}u_{i} & \text { if } s_{i}=(0,0), \\ \pi_{n_{i}} \cdot\left(1-u_{i}\right) & \text { if } m_{i}=1 .\end{cases}
$$

where $\pi_{n_{i}}$ is the stationary probability of a $M / M / \alpha / \alpha$ system such that

$$
\pi_{n_{i}}\left(\rho_{i}\right)=\frac{\left(\rho_{i}\right)^{n_{i}} / n_{i} !}{\sum_{n=0}^{\alpha}\left(\rho_{i}\right)^{n} / n !}, \forall n_{i} .
$$

Then, revenue and eviction cost occurs only when channel is idle because $n_{i}=0$ for a busy channel. Hence, for $m_{i}=1$, we derive the revenue rate $R\left(p_{i}, \rho_{i}\right)$ (the average revenue per unit time) and the eviction cost $E\left(p_{i}, \rho_{i}\right)$ as follows:

$$
\begin{aligned}
& R\left(p_{i}, \rho_{i}\right)=\sum_{n_{i}=0}^{\alpha} p_{i} n_{i} \cdot \pi_{n_{i}}\left(\rho_{i}\right)=p_{i} \rho_{i} \frac{\sum_{n=0}^{\alpha-1}\left(\rho_{i}\right)^{n} / n !}{\sum_{n=0}^{\alpha}\left(\rho_{i}\right)^{n} / n !} \\
& E\left(p_{i}, \rho_{i}\right)=\sum_{n_{i}=0}^{\alpha} I n_{i} \cdot \pi_{n_{i}}\left(\rho_{i}\right) \cdot \lambda_{i}^{O F F}=\beta \frac{\lambda_{i}^{O F F}}{\mu} R\left(p_{i}, \rho_{i}\right)
\end{aligned}
$$

where $\lambda_{i}^{O F F}$ is the transition probability due to the $\mathrm{OFF} \rightarrow \mathrm{ON}$ transition of an idle channel.

The derived $R\left(p_{i}, \rho_{i}\right)$ and $E\left(p_{i}, \rho_{i}\right)$ will become our bases in the later sections to calculate the profit rate of WSP $i$ under the various market conditions. For the ease of notation, we define

$$
\Delta\left(p_{i}, \rho_{i}\right):=R\left(p_{i}, \rho_{i}\right)-E\left(p_{i}, \rho_{i}\right) .
$$

\section{Price Competition Analysis}

In this section, we investigate a price competition subgame to find the best tariff strategy of WSP $i$ in terms of $p_{i}$, when $\left(u_{i}, u_{-i}\right)$ are given. We also study the necessary condition for the existence of the price NE and derive the NE at such conditions. The derived price NE will be applied to the quality full-game in Section VI, in determining the profit at the equilibrium price for a given set of quality.

\section{A. Three Pricing Strategies}

At WSP $i$, the price of the competitor WSP $-i$ (i.e., $p_{-i}$ ) is known since the WSP $-i$ advertises its tariff via the beacons. Then, the WSP $i$ can take one of the following three pricing strategies according to the relationship between $p_{i}$ and $p_{-i}$ : 
(1) $p_{i}<p_{-i}$, (2) $p_{i}>p_{-i}$, and (3) $p_{i}=p_{-i}$. We overview each strategy to derive the conditional profit of WSP $i$.

- Under strategy $1\left(p_{i}<p_{-i}\right)$ : WSP $i$ can monopolize the market and hence its profit is maximized at $p_{i}=p_{-i}-\epsilon$, $\epsilon>0$, where $\epsilon$ can be arbitrarily small. In this case, the profit rate of WSP $i$ becomes

$$
F_{i}^{\left\{p_{i}<p_{-i}\right\}}=\left(1-u_{i}\right) \cdot \Delta\left(p_{-i}-\epsilon, \rho\right)-L_{i} .
$$

- Under strategy $2\left(p_{i}>p_{-i}\right)$ : WSP $i$ loses the entire market to its competitor (i.e., $\lambda_{i}=0$ and $\lambda_{-i}=\lambda$ ) if WSP $-i$ has an idle channel (i.e., $m_{-i}=1$ ). On the contrary, while $m_{-i}=0$, it becomes $\lambda_{i}=\lambda$ and $\lambda_{-i}=0$ if WSP $i$ 's channel is idle. Therefore, with probability $u_{-i} \cdot\left(1-u_{i}\right)$, the stationary probability of WSP $i$ 's system follows Eq. (3). In this case, WSP $i$ 's profit is maximized at $p_{i}=\bar{\Pi}$ since WSP $-i$ is out-of-service, and thus WSP $i$ 's profit rate is given as

$$
F_{i}^{\left\{p_{i}>p_{-i}\right\}}=\left(1-u_{i}\right) u_{-i} \cdot \Delta(\bar{\Pi}, \rho)-L_{i} .
$$

- Under strategy $3\left(p_{i}=p_{-i}\right)$ : Here, we need to consider two cases. First, when $m_{i}=m_{-i}=1$, two WSPs take an equal share of the market such that $\lambda_{i}=\lambda_{-i}=\lambda / 2$. Second, when $m_{i}=1$ and $m_{-i}=0$, we have $\lambda_{i}=\lambda$ and $\lambda_{-i}=0$ since the arriving customers cannot find the service beacons of WSP $-i$. Therefore, by setting $p_{i}=p_{-i}$, the profit rate of WSP $i$ becomes

$$
\begin{aligned}
F_{i}^{\left\{p_{i}=p_{-i}\right\}}=\left(1-u_{i}\right)\{ & u_{-i} \cdot \Delta\left(p_{-i}, \rho\right)+ \\
& \left.\left(1-u_{-i}\right) \cdot \Delta\left(p_{-i}, \frac{\rho}{2}\right)\right\}-L_{i} .
\end{aligned}
$$

\section{B. Optimal Price Strategy}

The goal of WSP $i$ is to maximize its profit by optimally determining its price $p_{i}$ for a given $p_{-i}$. Hence, we will compare the profit rates of the three pricing strategies in Section V-A to derive the optimal $p_{i}$.

1) Comparison of price strategies 1 and 3: We first compare strategies 1 and 3 in terms of their profit rates given by Eqs. (4) and (6) as follows (when $\epsilon \rightarrow 0^{+}$):

$$
\begin{aligned}
& F_{i}^{\left\{p_{i}<p_{-i}\right\}}-F_{i}^{\left\{p_{i}=p_{-i}\right\}}= \\
&\left(1-u_{i}\right)\left(1-u_{-i}\right) p_{-i}\left(1-\beta \frac{\lambda_{i}^{O F F}}{\mu}\right) A_{r}
\end{aligned}
$$

where

$$
A_{r}:=\rho\left\{\frac{\sum_{n=0}^{\alpha-1} \rho^{n} / n !}{\sum_{n=0}^{\alpha} \rho^{n} / n !}-\frac{1}{2} \cdot \frac{\sum_{n=0}^{\alpha-1}(\rho / 2)^{n} / n !}{\sum_{n=0}^{\alpha}(\rho / 2)^{n} / n !}\right\} .
$$

Lemma 1. For $0<\rho<1$ and $\alpha \geq 1, A_{r}>0$.

Proof: See Appendix A.

Theorem 1. Price strategy $3\left(p_{i}=p_{-i}\right)$ is strictly dominated by price strategy $1\left(p_{i}<p_{-i}\right)$.

Proof: In Eq.(7), $\left(1-\beta \lambda_{i}^{O F F} / \mu\right)>0$ since $\beta \leq 1$ and $\lambda_{i}^{O F F} / \mu<1$ by our assumption, and $A_{r}>0$ by Lemma 1 .
Therefore, we have $F_{i}^{\left\{p_{i}<p_{-i}\right\}}>F_{i}^{\left\{p_{i}=p_{-i}\right\}}, \forall p_{-i}$, which completes the proof.

2) Comparison of price strategies 1 and 2: Next, we compare strategies 1 and 2 in terms of their profit rates given by Eqs. (4) and (5) as follows (when $\epsilon \rightarrow 0^{+}$):

$$
\begin{aligned}
& F_{i}^{\left\{p_{i}<p_{-i}\right\}}-F_{i}^{\left\{p_{i}>p_{-i}\right\}}= \\
& \quad\left(1-u_{i}\right)\left(p_{-i}-u_{-i} \bar{\Pi}\right)\left(1-\beta \frac{\lambda_{i}^{O F F}}{\mu}\right) \frac{\rho \sum_{n=0}^{\alpha-1} \rho^{n} / n !}{\sum_{n=0}^{\alpha} \rho^{n} / n !} .
\end{aligned}
$$

Theorem 2. The optimal price strategy is

- Strategy $1\left(p_{i}=p_{-i}-\epsilon\right)$, if $p_{-i}>u_{-i} \cdot \bar{\Pi}$,

- Strategy $2\left(p_{i}=\bar{\Pi}\right)$, if $p_{-i} \leq u_{-i} \cdot \bar{\Pi}$.

Proof: By Theorem 1, price strategy 3 cannot be optimal. Hence, we only need to compare strategies 1 and 2 . In the proof of Theorem 1, it has been shown that $\left(1-\beta \lambda_{i}^{O F F} / \mu\right)>$ 0 . Therefore, by considering the form of Eq. (8), it is seen that strategy 1 is optimal (i.e., $F_{i}^{\left\{p_{i}<p_{-i}\right\}}>F_{i}^{\left\{p_{i}>p_{-i}\right\}}$ ) if $p_{-i}>u_{-i} \cdot \bar{\Pi}$; otherwise strategy 2 is optimal.

\section{Nash Equilibrium of the Price Competition}

Using the derived optimal price strategy, we can find the Nash Equilibrium of the price competition. We first describe how to determined the marginal price for each WSP, and then derive the NE and its existence condition.

1) Finding the Marginal Price: We define the marginal price $\underline{\Pi}_{i}$ as the minimal price to guarantee a non-negative profit for WSP $i$ even at the worst case. That is, when $p_{i}=\underline{\Pi}_{i}$, the WSP $i$ should achieve at least a break-even (zero profit) regardless of $p_{-i}$. When we fix $p_{i}=\Pi_{i}$, the profit rate $F_{i}$ previously given as Eqs. (4),(5),(6) becomes

- $F_{i}^{\left\{p_{i}<p_{-i}\right\}}=\left(1-u_{i}\right) \cdot \Delta\left(\underline{\Pi}_{i}, \rho\right)-L_{i}$,

- $F_{i}^{\left\{p_{i}>p_{-i}\right\}}=\left(1-u_{i}\right) \cdot u_{-i} \Delta\left(\underline{\Pi}_{i}, \rho\right)-L_{i}$,

- $F_{i}^{\left\{p_{i}=p_{-i}\right\}}=\left(1-u_{i}\right)\left\{u_{-i} \Delta\left(\underline{\Pi}_{i}, \rho\right)+\left(1-u_{-i}\right) \Delta\left(\underline{\Pi}_{i}, \frac{\rho}{2}\right)\right\}$ $-L_{i}$.

It can be observed that $F_{i}^{\left\{p_{i}>p_{-i}\right\}}$ is the worst. Therefore, to guarantee $F_{i} \geq 0$, we need to set

$$
\begin{aligned}
\underline{\Pi}_{i} & =\frac{L_{i} / u_{-i}}{\left(1-u_{i}\right) \cdot \Delta\left(\underline{\Pi}_{i}, \rho\right) / \underline{\Pi}_{i}}=\frac{L_{i} / u_{-i}}{\left(1-u_{i}\right) \chi_{i}(\rho)} \\
& =\frac{\bar{\gamma}_{1}\left(2-u_{i}-u_{-i}\right)^{\gamma_{2}}}{u_{-i} \chi_{i}(\rho)},
\end{aligned}
$$

where Eq.(1) is applied. Here, $\chi_{i}\left(\rho_{i}\right)$ is defined as

$$
\chi_{i}\left(\rho_{i}\right):=\left(1-\beta \frac{\lambda_{O F F}^{i}}{\mu}\right) \rho_{i} \frac{\sum_{n=0}^{\alpha-1}\left(\rho_{i}\right)^{n} / n !}{\sum_{n=0}^{\alpha}\left(\rho_{i}\right)^{n} / n !} .
$$

Note that if the determined $\underline{\Pi}_{i}$ satisfies $\underline{\Pi}_{i}>\bar{\Pi}$, WSP $i$ cannot make positive profit for any $p_{i}$ since $\lambda_{i}=0$ for $p_{i}>\bar{\Pi}$ making the profit strictly negative (i.e., $F_{i}=-L_{i}$ ). Therefore, WSP $i$ should opt out of the market when $\underline{\Pi}_{i}>\bar{\Pi}$ in order not to incur any channel leasing cost. In the next section, this will be modeled as forcing $u_{i}=1$ at the quality competition where the channel leasing cost becomes zero since $C_{i}^{e f f}=0$. 


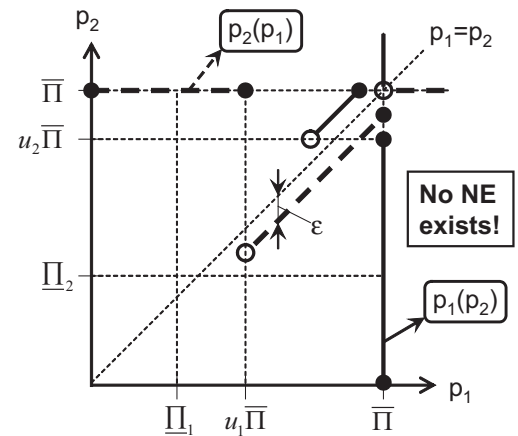

(a) $\underline{\Pi}_{1} \leq u_{1} \bar{\Pi}, \underline{\Pi}_{2} \leq u_{2} \bar{\Pi}$

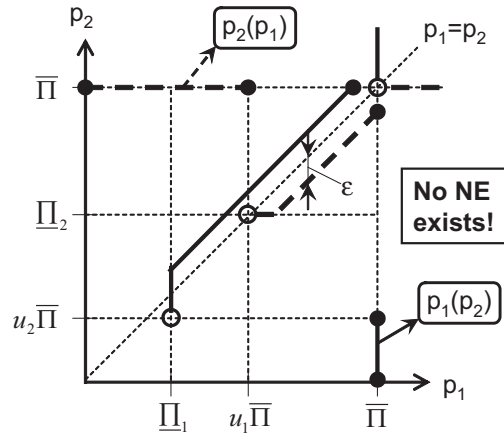

(b) $\underline{\Pi}_{1} \leq u_{1} \bar{\Pi}, \underline{\Pi}_{2}>u_{2} \bar{\Pi}$

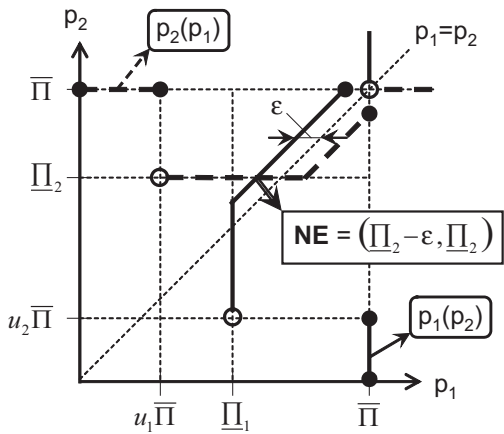

(c) $\underline{\Pi}_{1}>u_{1} \bar{\Pi}, \underline{\Pi}_{2}>u_{2} \bar{\Pi}$

Fig. 5. Nash Equilibrium of the price competition

2) Finding the Nash Equilibrium: To find the NE, we consider the case where $\underline{\Pi}_{i} \leq \bar{\Pi}$ and $\underline{\Pi}_{-i} \leq \bar{\Pi}$ so that both WSPs may participate in the market competition. Fig. 5 shows the NE of the price competition, where the solid plot represents $p_{1}\left(p_{2}\right)$, i.e., the best response function of WSP 1 given $p_{2}$, and the dashed plot represents $p_{2}\left(p_{1}\right)$. The best response functions are drawn by following the optimal strategy in Theorem 2 . Note that WSP $i$ never decreases its price $p_{i}$ smaller than $\underline{\Pi}_{i}$, and hence $p_{i}\left(p_{-i}\right)$ is lower-bounded by $\underline{\Pi}_{i}$.

It can be seen from Figs. 5(a),(b) that there exists no NE when (1) $\underline{\Pi}_{i} \leq u_{i} \bar{\Pi}$ and $\underline{\Pi}_{-i} \leq u_{-i} \bar{\Pi}$, or (2) $\underline{\Pi}_{i} \leq u_{i} \bar{\Pi}$ and $\underline{\Pi}_{-i}>u_{-i} \bar{\Pi}$, since $p_{1}\left(p_{2}\right)$ and $p_{2}\left(p_{1}\right)$ never intersect with each other.

As shown in Fig. 5(c), the NE exists when $\underline{\Pi}_{i}>u_{i} \bar{\Pi}$ and $\underline{\Pi}_{-i}>u_{-i} \bar{\Pi}$, where the NE is given as $\left(\underline{\Pi}_{2}-\epsilon, \underline{\Pi}_{2}\right)$ for $\underline{\Pi}_{2}>\underline{\Pi}_{1}$. Due to symmetry, the NE becomes $\left(\underline{\Pi}_{1}, \underline{\Pi}_{1}-\epsilon\right)$ for $\underline{\Pi}_{1}>\underline{\Pi}_{2}$. Therefore, as $\epsilon \rightarrow 0$, there exist a unique NE described as follows in Theorem 3.

Theorem 3. The NE of the price competition exists only when $\underline{\Pi}_{i}>u_{i} \bar{\Pi}$ and $\underline{\Pi}_{-i}>u_{-i} \bar{\Pi}$, and the unique NE is determined as $\left(p_{1}, p_{2}\right)=\left(p^{*}, p^{*}\right)$, where $p^{*}=\max \left\{\underline{\Pi}_{1}, \underline{\Pi}_{2}\right\}$ and $\underline{\Pi}_{i}$ is given as in Eq.(9).

\section{Quality COMPETITION ANALYSis}

The goal of the quality competition is to find the best channel to lease with optimal quality in terms of $u_{i}$ that achieves maximal profit of WSP $i$ at the equilibrium price found in Section V. Having the NE of the price competition as $p_{i}=p_{-i}=\max \left\{\underline{\Pi}_{1}, \underline{\Pi}_{2}\right\}$, the resulting profit of WSPs is given as Eq. (6), and we want to find the best response function $u_{i}\left(u_{-i}\right)$ to maximize such profit.

As introduced in the channel model, $u_{i}=\lambda_{i}^{O F F} /\left(\lambda_{i}^{O F F}+\right.$ $\left.\lambda_{i}^{O N}\right)$, and thus there exist infinitely many possible pairs of $\left(\lambda_{i}^{O F F}, \lambda_{i}^{O N}\right)$ for a given $u_{i}$. Therefore, we would like to consider a scenario where $\lambda_{i}^{O F F}=\lambda^{O F F}, \forall i$, representing the case when channels have identically distributed intervals between PU activities. It is also possible to consider another scenario where $\lambda_{i}^{O N}=\lambda^{O N}, \forall i$ (i.e., the duration of PU activities follows the same distribution over channels), which is left as our future work.
Note that even though the optimal $u_{i}$ can be found for any $\gamma_{2} \geq 1$, we are particularly interested in the case of $\gamma_{2}=1$ as an illustrative example.

\section{A. Market entry barrier}

WSP $i$ 's profit becomes strictly negative in case its marginal price becomes greater than the monopoly price (i.e., $\underline{\Pi}_{i}>\bar{\Pi}$ ), because there will be no customer arrival while the channel leasing fee must be still paid. If this happens, the WSP would rather shut down its service by leasing no channel (equivalently, leasing a channel with $u_{i}=1$ ). Therefore, there exists a market entry condition for a WSP, which is described by $\underline{\Pi}_{i} \leq \bar{\Pi}$. For a given $u_{-i}$, this condition results in the following interval of $u_{i}$ :

$$
2-\left(1+\frac{\bar{\Pi} \chi_{i}(\rho)}{\bar{\gamma}_{1}}\right) u_{-i} \leq u_{i} .
$$

In addition, the same claim applies to WSP $-i$ by switching the role of $i$ and $-i$.

\section{B. Region-specific optimal quality strategies}

According to the market entry points, the area of $\left(u_{1}, u_{2}\right)$ can be divided into five regions shown in Fig. 6, where $2 u^{A}$ can be either smaller or larger than 1 , without exceeding 2 .

1) Region I and II (below the market entry barrier): The region $\mathrm{I}$ is a rectangular area where $0 \leq u_{i} \leq 1$ and $0 \leq u_{-i}<u^{A}$. The region represents the case when WSP $i$ cannot overcome the market entry barrier and it leaves the market. Therefore, the best response function in $u_{-i} \in\left[0, u^{A}\right)$ is $u_{i}\left(u_{-i}\right)=1$.

The region II, that does not include the line $u_{i}=2-$ $\left(1+\bar{\Pi} \chi_{i}(\rho) / \bar{\gamma}_{1}\right) u_{-i}$, also belongs to the case when WSP $i$ cannot overcome the market entry barrier. However, the region does not include $u_{i}=1$, and hence no possible solution exists. As a result, we can ignore this region in deriving $u_{i}\left(u_{-i}\right)$.

2) Region III (a monopoly market): The region III includes the line $2-\left(1+\bar{\Pi} \chi_{i}(\rho) / \bar{\gamma}_{1}\right) u_{-i}=u_{i}$, but excludes the line $u_{-i}=2-\left(1+\bar{\Pi} \chi_{-i}(\rho) / \bar{\gamma}_{1}\right) u_{i}$. Therefore, $p_{-i}>\bar{\Pi}$ in the region due to the market entry condition, and thus WSP $-i$ will not have any customer arrival. As a result, WSP $i$ 


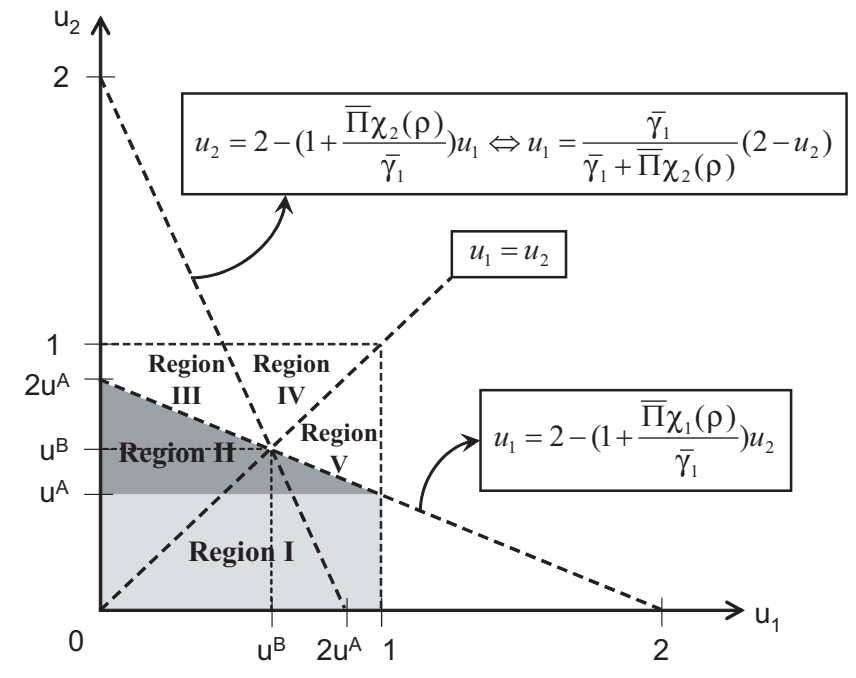

Fig. 6. Five decision regions of $\left(u_{1}, u_{2}\right)$

monopolizes the market with $p_{i}=\bar{\Pi}$. With the monopoly price, WSP $i$ 's profit rate becomes

$$
F_{i}^{I I I}=\left(1-u_{i}\right) \times\left\{\bar{\Pi} \chi_{i}(\rho)-\bar{\gamma}_{1}\left(2-u_{-i}-u_{i}\right)\right\}
$$

3) Region IV (a duopoly market): In the region IV, both WSPs can enter the market, and satisfy the condition $u_{i} \leq u_{-i}$ where we have $\underline{\Pi}_{i} \leq \underline{\Pi}_{-i}$. Therefore, the NE price becomes $p^{*}=\underline{\Pi}_{-i}$ and WSP $i$ 's profit rate becomes

$$
\begin{aligned}
F_{i}^{I V}= & \left(1-u_{i}\right) \times\left\{\left(u_{-i} \chi_{i}(\rho)+\left(1-u_{-i}\right) \chi_{i}(\rho / 2)\right) p^{*}\right. \\
& \left.-\bar{\gamma}_{1}\left(2-u_{-i}-u_{i}\right)\right\} \\
= & \bar{\gamma}_{1}\left(1-u_{i}\right)\left(2-u_{-i}-u_{i}\right)\left(\psi-u_{i}\right) \cdot \frac{1}{u_{i}},
\end{aligned}
$$

where $\psi=u_{-i}+\left(1-u_{-i}\right) \chi_{i}(\rho / 2) / \chi_{i}(\rho)$. For $u_{i} \in$ $\left[\frac{\bar{\gamma}_{1}}{\bar{\gamma}_{1}+\overline{\bar{\Pi}} \chi_{-i}(\rho)}\left(2-u_{-i}\right), u_{-i}\right]$, Eq. (13) is maximized at $u_{i}=$ $\frac{\bar{\gamma}_{1}\left(\bar{\gamma}_{1}+\bar{\Pi} \chi_{-i}(\rho)\right.}{}\left(2-u_{-i}\right)$, because $F_{i} \rightarrow \infty$ as $u_{i} \rightarrow 0$ and $u_{-i} \leq \psi<1 \leq\left(2-u_{-i}\right)$ where $\psi<1$ is given by Lemma 1 .

4) Region $V$ (a duopoly market): Similar to the region IV, the region $\mathrm{V}$ also belongs to the duopoly market. As seen, the region satisfies the condition $u_{i} \geq u_{-i}$ where we have $\underline{\Pi}_{i} \geq \underline{\Pi}_{-i}$. Therefore, the NE price becomes $p^{*}=\underline{\Pi}_{i}$, and WSP $i$ 's profit rate becomes

$$
F_{i}^{V}=\left(u_{i}-1\right)\left(u_{i}+u_{-i}-2\right) \cdot \frac{\bar{\gamma}_{1}\left(1-u_{-i}\right) \chi_{i}(\rho / 2)}{u_{-i} \chi_{i}(\rho)} .
$$

Then, due to the form of the region $\mathrm{V}$, two distinct cases are considered. First, for $u_{-i} \in\left[u^{A}, u^{B}\right)$, we have $0<2-$ $\left(1+\bar{\Pi} \chi_{i}(\rho) / \bar{\gamma}_{1}\right) u_{-i} \leq u_{i} \leq 1$. Since $1 \leq\left(2-u_{-i}\right)$, Eq. (14) is maximized at $u_{i}=2-\left(1+\bar{\Pi} \chi_{i}(\rho) / \bar{\gamma}_{1}\right) u_{-i}$.

Next, for $u_{-i} \in\left[u^{B}, 1\right]$, we have $u_{i} \in\left[u_{-i}, 1\right]$, and thus Eq. (14) is maximized at $u_{i}=u_{-i}$.

\section{Nash Equilibrium of the Quality Competition}

Finally, we derive the optimal quality strategy, i.e., the best response function $u_{i}\left(u_{-i}\right)$ for all possible intervals of $u_{-i}$. As already shown, the best response function in $u_{-i} \in\left[0, u^{A}\right)$ is

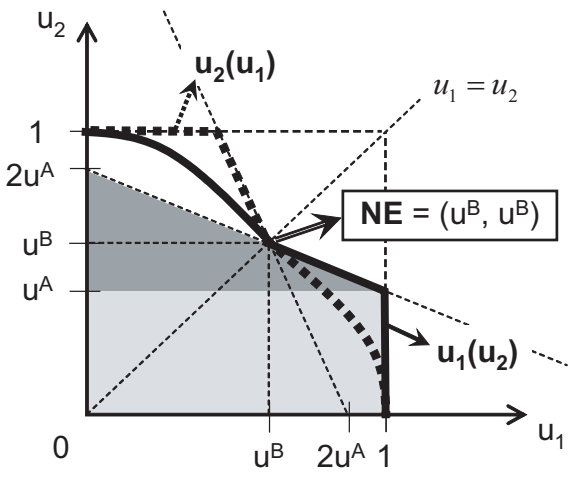

Fig. 7. The Nash Equilibrium of the quality competition

$u_{i}\left(u_{-i}\right)=1$. For $u_{-i} \in\left[u^{A}, u^{B}\right)$, we compare the regions II and V, but as pointed out earlier, we can ignore the region II. Therefore, the best response function in $u_{-i} \in\left[u^{A}, u^{B}\right)$ is $u_{i}\left(u_{-i}\right)=2-\left(1+\bar{\Pi} \chi_{i}(\rho) / \bar{\gamma}_{1}\right) u_{-i}$.

For $u_{-i} \in\left[u^{B}, 1\right]$, we need to compare all three regions (III, $\mathrm{IV}$, and $\mathrm{V}$ ) in order to determine the best response function. First, it can be easily shown that the best profit in the region V cannot exceed the best profit of the region IV for the following reason. The maximal profit of the region $\mathrm{V}$ for $u_{-i} \in\left[u^{B}, 1\right]$ is achieved at $u_{i}=u_{-i}$, which is a shared line with region IV. However, $u_{i}=u_{-i}$ does not provide the maximal profit in the region IV, and thus the maximal profit of the region IV is larger than that of the region $\mathrm{V}$.

Next, for given $u_{-i}$, let $u_{i}^{I V}$ denote the optimal $u_{i}$ in the region IV such as $u_{i}^{I V}=\frac{\bar{\gamma}_{1}}{\bar{\gamma}_{1}+\bar{\Pi} \chi_{-i}(\rho)}\left(2-u_{-i}\right)$. Let us also consider $u_{i}=u_{i}^{I V}-\epsilon, \epsilon>0$, which is in the region III. If we compare Eq. (13) at $u_{i}=u_{i}^{I V}$ and Eq. (12) at $u_{i}=$ $u_{i}^{I V}-\epsilon$ for an arbitrarily small $\epsilon$, then we can easily observe that the leasing cost is arbitrarily close to each other in both regions while $\bar{\Pi} \chi_{i}(\rho)>\left(u_{-i} \chi_{i}(\rho)+\left(1-u_{-i}\right) \chi_{i}(\rho / 2)\right) \underline{\Pi}_{-i}$. Therefore, we can conclude that the best profit in the region IV cannot exceed the profit in the region III at $u_{i}=u_{i}^{I V}-\epsilon$, and thus the best response function exists in the region III.

Fig. 7 plots the resulting best response $u_{i}\left(u_{-i}\right)$ for $u_{-i} \in$ $[0,1]$, where $u_{-i}\left(u_{i}\right)$ is also drawn using the symmetry. It is observed that, regardless of the optimal $u_{i}$ in the region III, there exists a NE of the quality game at $\left(u^{B}, u^{B}\right)$ where $u^{B}$ is an intersection of $u_{i}=u_{-i}$ and $u_{i}=2-$ $\left(1+\bar{\Pi} \chi_{i}(\rho) / \bar{\gamma}_{1}\right) u_{-i}$, resulting in Theorem 4 as follows.

Theorem 4. The NE of the quality competition exists at $\left(u_{1}, u_{2}\right)=\left(u^{*}, u^{*}\right)$, where $u^{*}=2 \bar{\gamma}_{1} /\left\{2 \bar{\gamma}_{1}+\bar{\Pi} \chi_{1}(\rho)\right\}$.

Note that there exist two additional NEs other than $\left(u_{1}, u_{2}\right)=\left(u^{*}, u^{*}\right):(\tilde{u}, 1)$ and $(1, \tilde{u})$, where $0 \leq \tilde{u}<u^{A}$. However, according to the concept of the focal point introduced in [13], [20], these NEs are not likely to be chosen by the WSPs since such NEs exclude either WSP from the market thus impairing the fairness.

From Theorem 4, we obtain the following two corollaries.

Corollary 1. At the NE of the quality competition, the $N E$ 
price becomes $p^{*}=\bar{\Pi}$.

Proof: By applying $u_{i}=u_{-i}=u^{*}$ to Eq. (9), we obtain $\underline{\Pi}_{i}=\bar{\Pi}$ since $\chi_{1}(\rho)=\chi_{2}(\rho)$. Therefore, $p^{*}=\bar{\Pi}$ by Theorem 3 .

Corollary 2. The NE of the quality competition satisfies the equilibrium price existence condition.

Proof: Since $\underline{\Pi}_{i}=\bar{\Pi}$, we have $\underline{\Pi}_{i}>u_{i} \bar{\Pi}$ and $\underline{\Pi}_{-i}>$ $u_{-i} \bar{\Pi}$ for $u_{i}=u_{-i}=u^{*} \neq 1$.

\section{Discussion}

WSPs may, in reality, not be able to find the channel that exactly matches their needs, whose utilization factor equals $u^{*}$. In such a case, they should make a reasonable assumption that both WSPs would act rationally to bid for the best matching channel whose utilization factor is closest to $u^{*}$. This strategy is reasonable in that the quality competition is a one-shot game performed once at each periodic auction, and thus a WSP cannot make any adjustment on its leased channel until the next auction.

In case $u$ takes its value from a countable set in a discrete manner, the quality competition becomes a combinatorial matching problem that should consider all possible pairs of $\left(u_{1}, u_{2}\right)$ from the given channel set. The problem formulation in such a case becomes quite different from the procedure presented in this paper, as the best response function is no more continuous. We leave the case as our future work.

The quality competition problem can be further extended to a joint quantity/quality competition when we consider WSPs, each operating with multiple channels. That is, WSP $i$ leases more than one channel, say $M_{i}$ channels, and combine them into a one logical channel with a larger capacity (e.g., $M_{i} \cdot C$ ). In such a case, we need to find the best quantity/quality pair of $\left(M_{i}, u_{i}\right),{ }^{8}$ for a given pair of $\left(M_{-i}, u_{-i}\right)$. Hence, the decision space becomes $\mathbb{N} \times[0,1]$ where $\mathbb{N}$ is a set of natural numbers, with additional complexity coming from the choice of $M_{i}$. We also leave such extension as our future work.

\section{EVAluation OF Wi-Fi 2.0 Network DyNAMics}

We now conduct an extensive numerical analysis to provide insight into the market dynamics of the Wi-Fi 2.0 network. First, we compare the profit observed from a simulated scenario with the analytically-derived profit to investigate the condition under which our state decomposition approach can be applied with a tolerable approximation error. Next, we study the fundamental tradeoffs between the network parameters at equilibrium, including the arrival rate $\lambda$, the leasing cost $\bar{\gamma}_{1}$, and the eviction cost $\beta$. In each scenario, we set a list of common parameters as follows: $C=5, B=1$, and $\gamma_{2}=1$.

\section{A. Approximation accuracy in state decomposition}

We compare the profit observed from the simulation with the profit given by the analysis to derive the condition under

\footnotetext{
${ }^{8}$ Assuming all $M_{i}$ channels are of the same quality $u_{i}$.
}

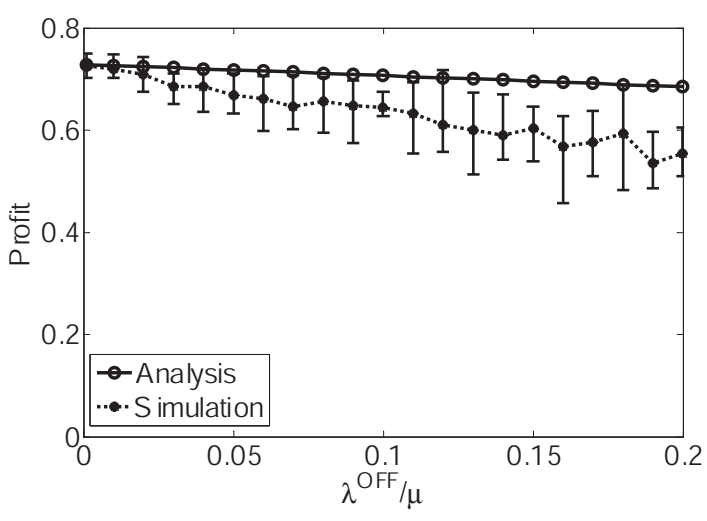

Fig. 8. Decomposition approximation accuracy

which state decomposition performs reasonably well. In the simulation, we randomly generate 200 pairs of exponential ON and OFF periods and also emulate user arrivals and departures. A simulation is run by applying the optimal price and quality found by the analysis, and repeats 10 times to derive the average performance. Other simulation parameters are set as $\bar{\Pi}=2, \rho=0.9, \bar{\gamma}_{1}=0.1$, and $\beta=0.25$.

From Fig. 8, one can see that the profit predicted by analysis gets fairly close to the actual achieved profit at a small $\lambda^{O F F} / \mu$, and the gap between them gradually increases as $\lambda^{O F F} / \mu$ grows. At $\lambda^{O F F} / \mu=0.1$, the approximation error is found to be less than $9.5 \%$, which becomes around $15 \%$ at $\lambda^{O F F} / \mu=0.15$. In case the tolerable error is less than $10 \%$, the state decomposition approach is effective for $\lambda^{O F F} / \mu \leq 0.1$, implying that an OFF period, on average, can accommodate at least 10 consecutive user sessions. Note that this is a plausible scenario since DSA targets to reuse underutilized channels with relatively larger ON/OFF periods than customer arrivals/departures.

\section{B. Impact of arrival rate and leasing cost}

In Fig. 9(a), we plot the achieved profit of a WSP at its NE while varying the arrival rate $\lambda$ (equivalently $\rho$ ). The leasing cost is also varied by testing three selected values of $\bar{\gamma}_{1}$. Other simulation parameters are set as $\bar{\Pi}=1, \mu=1 / 5, \lambda^{O F F}=$ $1 / 500, \lambda^{O N}=1 / 50$, and $\beta=0.25$. It can be seen that as the arrival rate increases (i.e., $\rho \rightarrow 1$ ), the WSP achieves more profit due to the increased revenue. The profit also enhances as $\bar{\gamma}_{1}$ decreases, due to the less leasing cost by $L_{i}$.

In Fig. 9(b), we plot the quality NE $u^{*}$ under the same test conditions. As the arrival rate increases, $u^{*}$ is monotonically decreasing because the WSP can overcome the leasing cost by accommodating more customers using a less busy channel (i.e., smaller $u^{*}$ ). Therefore, when $\rho \rightarrow 0$, the best strategy is to leave the market (i.e., $u^{*}=1$ ). On the other hand, at the same $\rho, u^{*}$ increases as $\bar{\gamma}_{1}$ increases, because it can compensate the increased leasing cost by using a less idle channel.

\section{Impact of eviction cost}

In Fig. 9(c), we plot the achieved profit at equilibrium versus the average $\mathrm{OFF}$ period (i.e., $1 / \lambda^{O F F}$ ) under the various 


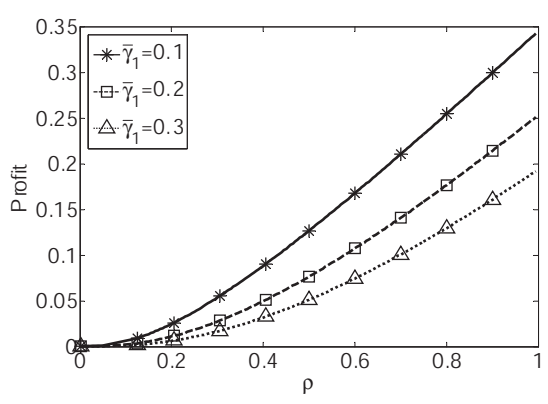

(a) Profit $F_{i}$ vs. arrival rate $\lambda$

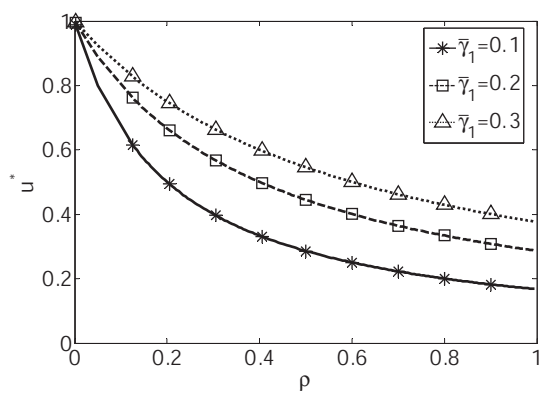

(b) Quality NE $u^{*}$ vs. arrival rate $\lambda$

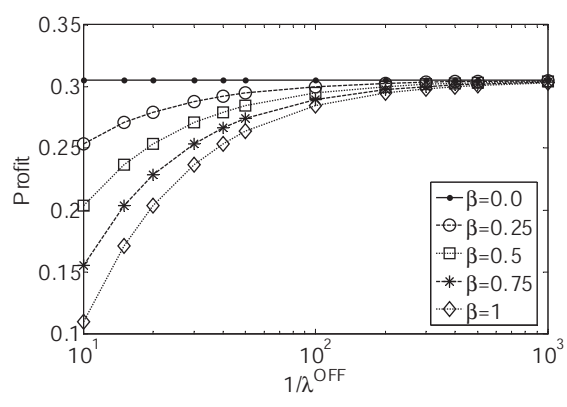

(c) Profit $F_{i}$ vs. eviction cost $\beta$

Fig. 9. Fundamental tradeoffs in the duopoly market

eviction costs given by $\beta$. Other simulation parameters are set as $\bar{\Pi}=1, \rho=0.9, \bar{\gamma}_{1}=0.3$, and $\lambda^{O N}=1 / 50$.

At the same $1 / \lambda^{O F F}$, a larger profit is achieved at a smaller $\beta$ due to the amount of reimbursement to the evicted users, and the difference becomes more pronounced as $1 / \lambda^{O F F}$ decreases. This implies that eviction becomes more dominant in a dynamic channel environment (i.e., channels with small $\mathrm{ON}$ and OFF periods) than in a static environment, due to more frequent evictions.

\section{CONCLUSION}

We studied the competition between WSPs in the duopoly Wi-Fi 2.0 network while considering time-varying spectrum availability. We modeled the problem as a joint price and quality game where two co-located WSPs compete for leasing limited spectrum resources and enticing customers to their service. A WSP's profit function is derived by considering the dependency of revenue on the pricing policy, the eviction cost due to the reimbursement of the evicted customers, and the channel leasing cost. It is shown that the price game has a unique NE at a larger marginal price of the two WSPs, and the quality game has a NE that balances the marginal price with the monopoly price. Using an extensive numerical analysis, we have demonstrated the fundamental tradeoffs in the Wi-Fi 2.0 network due to the factors, such as customer arrival rate, channel dynamics, and eviction cost.

In future, we would like to extend the problem by generalizing the number of WSPs, by considering multi-channel scenarios, and by accounting for other factors affecting the customer's preference in choosing a service such as QoS.

\section{REFERENCES}

[1] FCC. Second Report and Order and Memorandum Opinion and Order. FCC 08-260, November 2008.

[2] IEEE 802.22 Working Group on Wireless Regional Area Networks. http://www.ieee802.org/22/.

[3] S. Deb, V. Srinivasan, and R. Maheshwari. Dynamic Spectrum Access in DTV Whitespaces: Design Rules, Architecture and Algorithms. In ACM MobiCom, September 2009.

[4] P. Bahl, R. Chandra, T. Moscibroda, R. Murty, and M. Welsh. White Space Networking with Wi-Fi like Connectivity. In ACM SIGCOMM, August 2009.

[5] A. Stirling. White Spaces - the New Wi-Fi? International Journal of Digital Television, 1(1):69-83, 2010.

[6] H. Kim and K.G. Shin. Understanding Wi-Fi 2.0: From The Economical Perspective of Wireless Service Providers. IEEE Wireless Communications Magazine, pages 2-8, August 2010.
[7] J.M. Chapin and W.H. Lehr. The Path to Market Success for Dynamic Spectrum Access Technology. IEEE Communications Magazine, 45(5):96-103, May 2007.

[8] M.M. Buddhikot. Understanding Dynamic Spectrum Access: Models, Taxonomy and Challenges. In IEEE DySPAN, April 2007.

[9] H. Kim and K.G. Shin. Optimal Admission and Eviction Control of Secondary Users at Cognitive Radio HotSpots. In IEEE SECON, 2009.

[10] H. Kim and K.G. Shin. Efficient Discovery of Spectrum Opportunities with MAC-Layer Sensing in Cognitive Radio Networks. IEEE Transactions on Mobile Computing, 7(5):533-545, May 2008.

[11] S. Geirhofer, L. Tong, and B.M. Sadler. Dynamic Spectrum Access in the Time Domain: Modeling and Exploiting White Space. IEEE Communications Magazine, 45(5):66-72, May 2007.

[12] J. Jia and Q. Zhang. Competitions and Dynamics of Duopoly Wireless Service Providers in Dynamic Spectrum Market. In ACM MobiHoc, 2008.

[13] L. Duan, J. Huang, and B. Shou. Competition with Dynamic Spectrum Leasing. In IEEE DySPAN, April 2010.

[14] G. S. Kasbekar, E. Altman, and S. Sarkar. A Hierarchical Spatial Game over Licenced Resources. In GameNets, June 2009.

[15] G.S. Kasbekar and S. Sarkar. Spectrum Pricing Games with Bandwidth Uncertainty and Spatial Reuse in Cognitive Radio Networks. In $A C M$ MobiHoc, September 2010.

[16] Y. Wu, B. Wang, K.J.R. Liu, and T.C. Clancy. A Multi-Winner Cognitive Spectrum Auction Framework with Collusion-Resistant Mechanisms. In IEEE DySPAN, October 2008.

[17] S. Gandhi, C. Buragohain, L. Cao, H. Zheng, and S. Suri. A General Framework for Wireless Spectrum Auctions. In IEEE DySPAN, 2007.

[18] D. Niyato and E. Hossain. A Game-Theoretic Approach to Competitive Spectrum Sharing in Cognitive Radio Networks. In IEEE WCNC, 2007.

[19] S. Ghani and M. Schwartz. A Decomposition Approximation for the Analysis of Voice/Data Integration. IEEE Transactions on Communications, 42(7):2441-2452, July 1994.

[20] R.B. Myerson. Game Theory: Analysis of Conflict. Harvard University Press, Cambridge, MA, 2002.

\section{APPENDIX A}

PROOF OF LEMMA 1

$$
\begin{aligned}
& \frac{\sum_{n=0}^{\alpha} \rho^{n} / n !}{\sum_{n=0}^{\alpha-1} \rho^{n} / n !}-2 \cdot \frac{\sum_{n=0}^{\alpha}(\rho / 2)^{n} / n !}{\sum_{n=0}^{\alpha-1}(\rho / 2)^{n} / n !}=1+\frac{\rho^{\alpha} / \alpha !}{\sum_{n=0}^{\alpha-1} \rho^{n} / n !} \\
& -2 \cdot\left(1+\frac{(\rho / 2)^{\alpha} / \alpha !}{\sum_{n=0}^{\alpha-1}(\rho / 2)^{n} / n !}\right)=\frac{\rho^{\alpha} / \alpha !}{\sum_{n=0}^{\alpha-1} \rho^{n} / n !}-1-2 . \\
& \frac{(\rho / 2)^{\alpha} / \alpha !}{\sum_{n=0}^{\alpha-1}(\rho / 2)^{n} / n !}<0, \quad \text { since } \frac{\rho^{\alpha} / \alpha !}{\sum_{n=0}^{\alpha-1} \rho^{n} / n !}<1 \text { because }
\end{aligned}
$$

(1) $\rho^{\alpha} / \alpha$ ! $<1$, for $0<\rho<1, \alpha \geq 1$, and (2) $\sum_{n=0}^{\alpha-1} \rho^{n} / n$ ! = $1+\sum_{n=1}^{\alpha-1} \rho^{n} / n !>1$. This completes the proof. 